\title{
Generic Object Discrimination for Mobile Assistive Robots using Projective Light Diffusion
}

\author{
Panagiotis Papadakis ${ }^{1}$, David Filliat ${ }^{2}$ \\ ${ }^{1}$ IMT Atlantique Bretagne/Pays de la Loire, LabSTICC UMR 6285, team IHSEV, Plouzané, France \\ ${ }^{2}$ ENSTA ParisTech, U2IS, Inria FLOWERS team, Université Paris-Saclay, Palaiseau, France
}

\begin{abstract}
A number of assistive robot services depend on the classification of objects while dealing with an increased volume of sensory data, scene variability and limited computational resources. We propose using more concise representations via a seamless combination of photometric and geometric features fused by exploiting local photometric/geometric correlation and employing domain transform filtering in order to recover scene structure. This is obtained through a projective light diffusion imaging process (PLDI) which allows capturing surface orientation, image edges and global depth gradients into a single image. Object candidates are finally encoded into a discriminative, wavelet-based descriptor allowing very fast object queries. Experiments with an indoor robot demonstrate improved classification performance compared to alternative methods and an overall superior discriminative power compared to state-of-the-art unsupervised descriptors within ModelNet10 benchmark.
\end{abstract}

\section{Introduction}

In the development of assistive service robots for indoor operation, the capability to detect and recognize generic 3D objects is indispensable. The fact that a number of robot skills such as manipulation, semantic mapping and navigation are directly dependent on this capability can largely explain its prevalent role, which is further promoted by the advent of sensors that provide synchronized photometric and geometric (RGB Color and Depth) information. RGB-D sensors have boosted progress in the above research fields as they enable the parallel use of 3D geometric and 2D image processing for improving object perception.

The challenges in object perception are strongly related to the object classes that are expected to be encountered as well as their surroundings. For instance, in the case of robotic manipulation scenarios object texture is highly informative of the object class while sensor noise is reduced due to small object/sensor distance and stable environment lighting. Works that are concerned with object perception for robotic manipulation $[1,2]$ may frequently discount efficiency at the benefit of discriminative power through separate treatment of color and geometry.

Conversely, in the case of object perception during mapping $[3,4]$ shape becomes more discriminative than texture due to the increased sensor/object distance while lighting conditions can vary considerably. As a result, processing the entire volume of sensory data may hinder a timely robot response to certain object classes, therefore exploiting both color and geometry is generally more computationally restricting. The issue had been early recognized, nevertheless, more recent works [5] suggest that real-time as well as reliable object perception remains challenging.

Here we are concerned with the latter problem, namely, detection and classification of objects of interest in the surroundings of a robot during indoor mapping. The problem is conceptually related to numerous works in the domain of computer vision, e.g. [6],[7] and [8]. Nevertheless, relevant works with a clear robotic scope as e.g. in [9] are relatively sparse. This is largely due to the low resolution of RGBD data within a narrow field-of-view (FOV) and increased noise which restricts the application of more elaborate signal processing as it impacts real-time performance.

Some works such as [10], [11], seek to alleviate the effect of narrow FOV by employing RGB-D based SLAM in order to stitch together the corresponding object views. Contrarily to such approaches, our objective is to reduce the necessity for constructing global metric-scale maps and instead primarily rely on semantic maps where even partially observed objects are used as landmarks. Therefore, our starting point for object perception is to treat single RGBD scene frames rather than complete 3D object reconstructions. In corroboration to this, the large-scale experiments presented in [8] or [12] clearly suggest that view-based methods generally outmatch complete 3D-based shape description methodologies.

Finally, works which are based on learning dictionaries/Bag-of-Words [13], [14] or deep neural networks [15] are beyond our scope here, since we aim at 
studying the generalization capability of our representation and derive a fair evaluation among alternative representations, without requiring a large training dataset. In this work we are strictly focused on the design of informative shape representations and descriptions exploitable in a robotic context, not on potential fine-tuning/training that could be applied in the sequel regardless of the underlying representation. Characteristically, the latest evolution of state-of-the-art PANORAMA representation [16] introduced by [17] which couples its discrimination capacity with training in the form of convolutional neural networks was shown to outperform the state-of-the-art on synthetic 3D object discrimination.

The main contribution claimed by this work is a novel representation termed as projective light diffusion image (PLDI) which can encode surface orientation, depth as well as photometric characteristics that can be subsequently captured in concise and efficient to compute shape descriptors. Here, we perform proof-of-concept experiments by expanding PLDIs into a set of wavelet-based features by evolving earlier work [16], originally designed for generic 3D object discrimination. This results in a new descriptor based on PLDI that allows faster and more discriminative object matching when compared to competitive state-of-the-art approaches in common indoor scenarios, combined with a remarkably low feature dimensionality. This makes it particularly suited for robotic applications which often impose considerable constraints in terms of computational and storage capacity. Through the proposed approach, our robot was able to reliably discriminate various real objects using a total of only \#4 synthetic object templates per class.

We organize the remainder of the article as follows. In section 2 we unfold our approach for fusing photometric with geometric information to compensate for noisy or missing sensor readings. In section 3 we detail the extraction of projective light diffusion images and subsequently in sections 4 and 5, the object candidate segmentation and feature extraction/matching components of our methodology, respectively. Finally, section 6 evaluates the performance of the complete framework in experiments with a mobile robot and compares against related alternatives.

\section{Filtering}

Conventional RGB-D sensors provide $15 / 30 \mathrm{fps}$ at a maximum resolution of around $1 \mathrm{Mpixel}$ and depth images that are subject to intense noise levels. To alleviate the impact of noise in the sensory data we adopt an approach to fuse color with depth information into a single representation. Our motivation for color and depth fusion is two-fold; (i) to exploit local correlation existing between image intensities and 3D geometry for noise filtering and edge preservation and (ii) reduce the total processing time by subsequently treating a single fused representation for feature ex- traction instead of separate color and depth descriptors.

In choosing a fusion scheme, we first note that raw depth data are subject to considerably higher levels of noise in comparison to color [18]. This is mainly because the acquired depth images often contain large areas of invalid/unknown depth pixel values induced by nonreflective/transparent surfaces or limited FOV. This suggests exploiting the the 3-channel color image as a means for recovering the 1-channel depth information.

Towards this goal, we use the 4-channel image of an RGB-D frame as a filtering guide and employ three Gaussian kernels that operate within the color, depth and the 2D image domain space respectively. The operation resembles that of the conventional bilateral filtering but differentiates with respect to the input and desired output image, known as joint bilateral filtering (JBF) [19]. We employ JBF by treating the RGB-D image as a guide in order to obtain a reconstructed depth and ignore pixels with invalid depth values which would mislead filtering.

Let $I, J$ denote the raw 3-channel RGB image and 1channel depth (Fig. 1 (a)) respectively, of a given RGB-D frame and $G_{d}, G_{i}$, and $G_{j}$ the selected domain, color and depth Gaussian kernels respectively. The cross filtered image $\tilde{J}$ (Fig. 1 (b)) is obtained as:

$$
\begin{array}{r}
\tilde{J_{\mathbf{p}}}=\frac{1}{C_{\mathbf{p}}} \sum_{\mathbf{q} \in \Omega \mid \exists J_{\mathbf{q}}} G_{d}(\|\mathbf{p}-\mathbf{q}\|) G_{i}\left(I_{\mathbf{p}}-I_{\mathbf{q}}\right) G_{j}\left(J_{\mathbf{p}}-J_{\mathbf{q}}\right) J_{\mathbf{q}} \\
C_{\mathbf{p}}=\sum_{\mathbf{q} \in \Omega \mid \exists J_{\mathbf{q}}} G_{d}(\|\mathbf{p}-\mathbf{q}\|) G_{i}\left(I_{\mathbf{p}}-I_{\mathbf{q}}\right) G_{j}\left(J_{\mathbf{p}}-J_{\mathbf{q}}\right)
\end{array}
$$

where $\mathbf{p}, \mathbf{q} \in \Omega$ denote $2 \mathrm{D}$ pixel locations in the image domain $\Omega \subset \mathbb{Z}_{*}^{2}$, where $\mathbb{Z}_{*}$ denotes the set of non-negative integers and $C_{\mathbf{p}}$ is the normalization denominator.

Multiple iterations of eq. (1) are possible in order to extend depth reconstruction until there are no missing depth values, however this increases the risk of physically connecting disjoint surfaces as a consequence of smoothing.

To avoid the direct computation of eq. (1) which operates in 6D space we follow the idea of Domain Transform filtering (DTF) presented in [20]. The core notion underlying DTF resides in isometrically transforming the domain of a multi-channel image into a $1 \mathrm{D}$ domain wherein geodesic distances in the original image are preserved within the transformed signal. Performing filtering of the transformed image using a 1D kernel along vertical and horizontal passes rather than using kernels in the original space, reduces the computational complexity of filtering to linear time by encoding the original kernel parameters within the domain transform (cf. p.4 in [20]). For completeness, we recall the central equation used for performing the DFT $c t$ (.) of the multichannel RGB-D image $S$ where $S_{\mathbf{q}}=\left(I_{\mathbf{q}}, J_{\mathbf{q}}\right) \in\left\{[0,255]^{3} \times \mathbb{R}^{+}\right\}$, namely:

$$
c t(u)=\int_{0}^{u} 1+\left|S_{\mathbf{q}_{x}}^{\prime}\right|{ }_{1} d x
$$



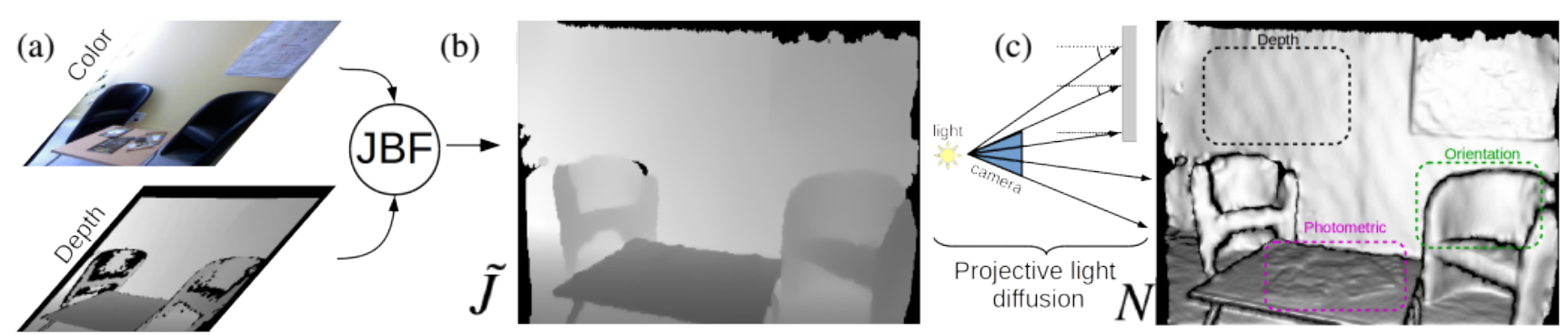

Figure 1. Filtering/fusion pipeline; (a) Raw depth image and RGB image, (b) reconstructed depth image and (c) projective light diffusion image. Emergent depth, photometric and surface orientation scene characteristics captured by PLDI are partially highlighted for emphasis (best viewed in color).

where $u$ corresponds either to the horizontal or the vertical image dimension depending on the filtering pass and $|\cdot|_{1}$ is the $L_{1}$ norm. Although more elaborate RGB-D filtering schemes such as [21] or [22] could be considered we advocate the use of JBF via DTF as being more suited for real-time applications without explicitly requiring a parallelized implementation. Furthermore, as our final goal is to classify $3 \mathrm{D}$ objects rather than recognize particular instances, recovering very subtle surface details is secondary if the main discriminative features of the observed objects are captured.

\section{Projective Light Diffusion Image Extraction}

Our shape description methodology relies mainly on the use of information about the surface orientation distribution of objects. The next step therefore amounts to the computation of the 3D surface orientation that is encoded within the filtered depth image. The procedure consists of three stages: (i) backward projection of depth pixels belonging to $\tilde{J}$ to the $3 \mathrm{D}$ coordinate frame of the sensor, (ii) computation of the 3D surface orientation tensor for the total set of reprojected points within the coordinate frame of the sensor and (iii) light source emulation at the center of the camera.

Letting $K$ be the camera matrix, we obtain the total set of 3D points as $P=\left\{\mathbf{v} \in \mathbb{R}^{3} \mid \mathbf{v}=K^{-1}\left[\mathbf{p}_{x}, \mathbf{p}_{y}, \tilde{J}_{\mathbf{p}}\right]^{T}, \mathbf{p} \in \Omega\right\}$ and the $3 \mathrm{D}$ surface orientation vectors as $\mathbf{n}_{\mathbf{p}}=\mathbf{e}_{2}$, where $\mathbf{e}_{0}, \mathbf{e}_{1}, \mathbf{e}_{2}$ correspond to the orthonormal eigenvectors of the local point density (within a maximum radius $r_{\text {max }}$ ), sorted in decreasing order. Fig. 1 (c) shows the output of this process denoted as image $N$, obtained by remapping in grayscale the cosine of the angle between normal vector $\mathbf{n}_{\mathbf{p}}$ and the camera projection ray $\mathbf{v}_{\mathbf{p}}$ passing through the respective pixel, namely:

$$
N_{\mathbf{p}}=\left|\mathbf{n}_{\mathbf{p}} \cdot \mathbf{v}_{\mathbf{p}}^{T}\right| /\left|\mathbf{v}_{\mathbf{p}}\right|
$$

The obtained image $N$ is hence the equivalent of the diffusion reflection component of light reflection models where in our case the light source coincides with the camera.

A qualitative comparison among initial raw depth/color data and corresponding PLDI reveals a number of advan- tages. Firstly, we note that previously unknown surface areas (mainly located in the interior of the sofas' boundaries, see Fig. 1 (a)) have now been largely recovered by using color and depth while preserving edge information. More importantly, new details originating from the image domain are emerging within the PLDI in predominantly planar areas (tabletop magazines, wall poster, floor tiles, wooden shelfside, blankets, see Figs 2 and 1 (c)). And finally, a new feature emerges due to the use of the projective light source emulation. In detail, while the 3D orientation of a surface itself does not convey any depth information locally, nevertheless, depth characteristics emerge globally for extended surfaces (mainly noticeable along walls in the provided examples and the double beds) as a result of the increasing intersection angle between the emulated light rays and the surface normals. PLDI is hence capable of jointly encoding surface orientation, depth and photometric features which proves advantageous is object classification compared to using separate images (see section 6).

\section{Object Clustering and Segmentation}

To segment candidate objects we impose a set of constraints that characterize (a) indoor scenes, (b) the robot sensing configuration and (c) the sensory data, following a paradigm similar to [9], [3] that assume moderate levels of clutter in terms of object/background interference. Our goal is to discriminate well separated from overly occluded candidates and attempt to recognize only the former. The latter could be treated at the decision level, e.g. through ac-

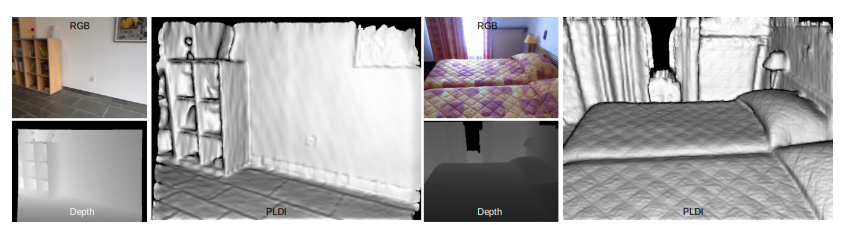

Figure 2. Example RGB-D scenes and corresponding PLDI images. Photometric features emerge along the floor (tiles), library (wood texture), poster et blanket covers. Depth gradients are noticeable along the wall and the double beds. 


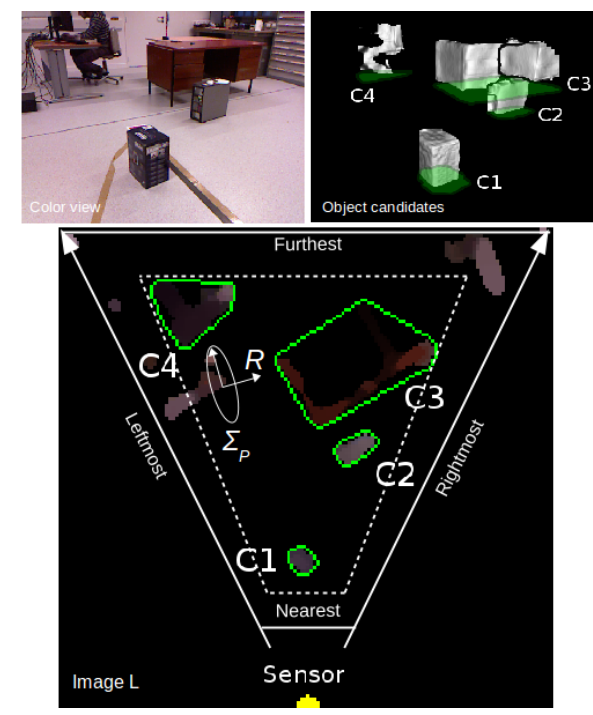

Figure 3. Object clusters detection and contours extraction. Density estimation is performed on image $L$ after aggregating the point projections to the floor plane. The sensory area corresponds to the interior of the dashed polygon.

tive perception in order to resolve the respective occlusion, which is beyond our scope here. On the contrary, clutter due to sensing noise is more amplified within our experimental scenario due to the variability of lighting conditions and extended perceptual range (see Sec. ??).

Indoor scene constraint We assume the existence of a dominant 2D plane which corresponds to the floor, serving as the lower boundary that splits in half the total 3D space and retains only parts that lie above. In practice, this plane corresponds to the configuration space used for pathplanning and can be easily obtained beforehand in the case where the sensor pose is fixed. For the case where the sensor pose changes with respect to the floor, we can update the parameters of the floor plane for every newly acquired $3 \mathrm{D}$ point cloud using robust estimation algorithms such as Random Sample Consensus (RANSAC).

Sensing constraints The robot sensing configuration is used to impose the remaining boundaries of the total sensory region. Two planes orthogonal to the floor are set for the furthest and nearest permissible distance from the sensor. Another two planes orthogonal to the floor that pass from the center of the sensor are used to the set the limits of the leftmost and rightmost permissible angles, set according to the corresponding leftmost and rightmost rays that emanate from the camera center. Setting an uppermost plane boundary is not required since the sensor is constantly directed towards the ground. The imposed limits are depicted in Fig. 3 for a top-down view of an example scene.

Data-driven constraints The set of RGB-D points $P_{S} \subset$ $P$ that lie within the constrained sensory area are subsequently aggregated by projecting them on the $2 \mathrm{D}$ floor

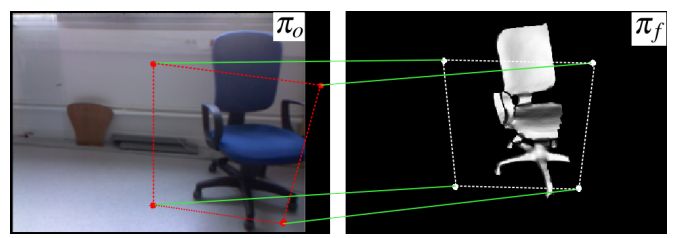

Figure 4. Perspective warping of segmented object from the projection plane $\pi_{o}$ defined between the actual object pose and camera coordinate frame, to the globally selected projection plane $\pi_{f}$.

plane, which produces an image denoted as $L \in \mathbb{Z}_{*}^{2}$ which is used as the domain where clustering is performed.

To obtain a set of object candidates, we apply an adaptive Parzen window density estimator using a 2D Gaussian kernel $G_{P}$ on image $L$ and next retain only those pixels with density higher than a threshold in order to filter out noisy measurements or small disjoint surfaces. Essentially, the chosen kernel accounts for the measurement error distribution of the camera sensor and depends on the angle between the pixel's camera ray and the camera's principal direction. The covariance matrix $\Sigma_{P}$ of $G_{P}$ is therefore obtained as $\Sigma_{P}=R^{T} \Sigma R$, where $R$ denotes the rotation matrix and $\Sigma$ the general measurement error covariance [18] (see Fig. 3). The retained pixel locations within $L$ are then grouped together by using conventional contour detection and border following. Fig. 3 shows an instance of a scene and the respective clustering (the exclusion of object clusters that are off the sensory boundaries can be observed in image $L$ ).

In the sequel, we perform a coarse ray tracing scan to determine if a cluster is potentially occluded and exclude such clusters in order to reduce classification errors. We perform ray tracing in the image $L$ for obtaining the pixels along a projection ray and for each such pixel assert whether it lies in the interior of an object contour. In the example of Fig. 3, clusters $C 3, C 4$ are finally excluded as other contours in the foreground intersect the corresponding rays.

Finally, we impose size constraints on the surface area within the contour of each cluster, i.e. the size of the $2 \mathrm{D}$ object footprint and the length of the contour and discard clusters that are excessively large or small. These thresholds are set according to the expected object classes.

Object clusters in image $L$ that successfully pass all the aforementioned tests are then used to segment the corresponding parts within image $N$ that are going to be used for feature extraction. The segmentation is performed by keeping only the $3 \mathrm{D}$ surface which lies within the boundaries of the respective contour of the footprint of each object, by applying depth culling within the depth image $\tilde{J}$.

As a final step, it is necessary to account for distortions that alter the projected shape of an object depending on the viewpoint. To address this issue, we choose a common projection plane $\pi_{f}$ that lies in front of the camera at the center of the chosen sensory area and then warp a candidate ob- 
ject image so that its actual projection plane $\pi_{o}$ matches the frontal plane $\pi_{f}$ using the respective homography (Fig. 4 illustrates a characteristic example). The $\pi_{o}$ plane parameters are obtained using the distance and angle between the camera frame and the centroid of the object.

A number of points are worth commenting after the completion this stage. In particular, the fact that an object may still be perspectively distorted after warping to $\pi_{f}$ is not problematic, since that distortion will be the same for all objects regardless of the viewpoint. In addition, the applied warping ensures that the distance from the sensor to the object has no effect on the scale of the PLDI of the object candidate. This means that if an object is observed from a distance which is further than the distance of $\pi_{f}$ from the sensor, then warping will have an upscaling effect on the initial PLDI and conversely, in the case where the object is observed from a nearest distance. Finally, it is true that while warping can account for the perspective distortion induced by the viewpoint, it does not warp the initial 3D rays $\mathbf{v}_{\mathbf{p}}$ used in eq. (3), to their new poses. Reapplying eq. (3) after warping, however, did not seem to have any noticeable impact in the resulting discrimination between objects.

\section{Features Representation}

Up to this point, we have segmented object candidates in the form of PLDIs which should be subsequently encoded through a corresponding feature-based description. Towards this direction, we integrate ideas from the stateof-the-art unsupervised 3D shape descriptor PANORAMA [16]. PANORAMA was originally designed for describing entire 3D polygonal models, it employs full 3D pose normalization, cylindrical projection extraction of separate depth and orientation images and a corresponding series expansion in space/time domain.

To accommodate differences existing with the current application domain, a number of adaptations were required. In detail, instead of having three orthogonal panoramic projections of an object aligned with its principal axes, here we only dispose a single view of the object that has been normalized to match the canonical projection plane. Hence, instead of relying on global object matching we rely on partial object matching via a single viewpoint since (as mentioned in Section 1) we seek to reduce the necessity for metric mapping and also because complete 3D object inspection is often impossible in reality. Most importantly, instead of using separate depth and surface orientation images as is done in PANORAMA, here we only use the PLDI image which is capable of encoding both modalities. We do this by retaining a wavelet-based decomposition of PLDI which is characterized by a high discriminative power/dimensionality ratio and a linear computational complexity through the use of the fast wavelet transform.

Letting $N: \mathbb{Z}_{*}^{2} \rightarrow[0,1]$ be the segmented and warped
PLDI of an object, we compute the corresponding 2D Discrete Wavelet Transform (DWT) expressed as:

$$
\begin{aligned}
W^{\phi}\left(j_{0}, m, n\right) & =\frac{1}{\sqrt{2 B \cdot 2 B}} \cdot \sum_{u=0}^{2 B-1} \sum_{v=0}^{2 B-1} N(u, v) \cdot \phi_{j_{0}, m, n}(u, v) \\
W^{\psi}(j, m, n) & =\frac{1}{\sqrt{2 B \cdot 2 B}} \cdot \sum_{u=0}^{2 B-1} \sum_{v=0}^{2 B-1} N(u, v) \cdot \psi_{j, m, n}(u, v)
\end{aligned}
$$

where $m, n \in[0,2 B-1], B$ is the sampling bandwidth, $j \geq j_{0}$ denotes the scale of the multi-level DWT, $j_{0}$ is the starting scale, $\phi_{j_{0}, m, n}, \psi_{j, m, n}$ are the scaling and wavelet functions respectively and $N(u, v)$ is the final segmented projective light diffusion image of a single object (e.g. Fig. 4 right). We compute a full-scale DWT of $\log _{2} 2 B$ levels, therefore $j=0,1, \ldots, \log _{2} 2 B-1$. The $W^{\phi}\left(j_{0}, m, n\right)$ approximation/scaling coefficients correspond to the lowpass subband of the transform at the starting scale $j_{0}$ while the $W^{\psi}(j, m, n)$ detail/wavelet coefficients correspond to the vertical, horizontal and diagonal subbands. We take the absolute value of the coefficients and normalize to their unit $L_{1}$ norm, which are now denoted as $\tilde{W}^{\phi}\left(j_{0}, m, n\right)$ and $\tilde{W}^{\psi}(j, m, n)$ (see [16] for further details).

Finally, we evaluate a set of central moments from the obtained coefficients image, namely, the mean, standard deviation and skewness, for each distinct sub-band and level $j>j_{0}$ of the transform (for the scale at the smallest resolution $j=j_{0}$ we keep the respective scalar values for the scaling and detail coefficients). The total procedure gives a final feature vector $\mathbf{f}$ of dimensionality $3 \times 3 \times\left(\log _{2} 2 B-1\right)+4$. The distance $d\left(\mathbf{f}_{1}, \mathbf{f}_{2}\right)$ between two feature vectors $\mathbf{f}_{1}, \mathbf{f}_{2}$ is evaluated by using the Canberra distance and object queries are appointed the class of the nearest neighbor from the sorted list of retrieved objects.

The main differences between the newly proposed PLDIbased descriptor and the original PANORAMA descriptor are summarized in the following table.

TABLE I. PLDI vs PANORAMA

\begin{tabular}{|l||c|c|}
\hline & PLDI & PANORAMA \\
\hline Partial 3D objects & $\checkmark$ & - \\
\hline Dimensionality & 58 & 15948 \\
\hline Features (Depth/Orientation/Photometric) & $\checkmark / \checkmark / \checkmark$ & $\checkmark / \checkmark /-$ \\
\hline
\end{tabular}

\section{Experiments}

To evaluate our approach we initially performed experiments in realistic conditions, elaborating on its effectiveness as well as efficiency aspects. Finally, we compare our method against state-of-the-art, unsupervised shape-based descriptors, both in realistic conditions as well as within a large-scale dataset and show that a significant advantage in discriminative power is attained. 


\subsection{Context}

The experiments were conducted for ROMEO2 project [23] which is a French National Project coordinated by SoftBank Robotics, formerly known as Aldebaran. The project aspires developing a state-of-the-art humanoid assistant robot for frail people and more generally for people with reduced mobility which can assist them in common daily tasks. This is made possible by developing advanced cognitive and operational skills, including, social humanrobot interaction, audiovisual sensing and object discovery and manipulation.

Among the various scenarios of interest, object perception is decomposed into two stages treated independently wherein the robot is primarily capable of perceiving and classifying large objects of interest placed on the ground (such as boxes, small and medium furniture, pots, etc) during navigation and secondarily, objects of interest lying on a table. The first stage is mainly exploitable for semantic mapping of the environment while the second is more pertinent to a subsequent object manipulation.

The following sections summarize the series of experiments that were performed in addressing the first stage, namely object discovery and classification of large objects using the Kinect v1 sensor by a mobile robot.

Ground-Truth The ground-truth used for the evaluation with a real robot is divided into two sets/splits of objects. The first set contains 3D object instances collected manually from the internet which belong to the classes of interest. We selected classes of 3D objects that were commonly encountered during indoor robot navigation and of fixed cardinality (4 objects per class). For each object, we then simulate a ring of $M$ viewpoints distributed at regular angular intervals around its centroid, at a fixed distance from the object ( 2 meters) and a camera pose equivalent to the configuration of the actual robot sensing system. In this manner, all objects are projected using the same reference plane $\pi_{f}$. Before projection, objects are placed along their expected orientation in the real world following a semi-automatic preprocessing step. Thus we finally obtain a synthesized set of $M$ PLDIs per object by simulating frame acquisitions of a Kinect sensor.

The second set contains the real 3D objects that were encountered by our mobile robot during exploration and that is used for testing the performance. In other words, whenever a real 3D object was segmented and deemed as a candidate as explained in Section 4, its corresponding PLDI descriptor is matched against all PLDI descriptors of the first set and it is appointed the class of the retrieved nearest neighbour.

The objects of the second set were encountered by a indoor robot equipped with an Kinect v1 sensor remotely operated in the interior of an office building. The visited areas included corridors, offices, lounge halls and a workshop, altogether characterized by a wide range of lighting condi- tions and scene/object configurations. Excluding corridors that were characterized by interior lighting and minimal object presence, all remaining areas where most objects were located were illuminated by strong exterior daylight thereby degrading the quality of depth acquisition in several cases. Finally, we note that since we match real 3D objects against synthetic 3D objects this induces a relatively high intraclass variance.

Table II summarizes the values used for the prominent parameters related to the evaluation in terms of the proposed method setting and the experiment.

TABLE II. Parameter setting \& Experiment

\begin{tabular}{|l|c|}
\hline Radii $G_{d}, G_{i}, G_{j}, r_{\max }$ & $12(p x), 10(R G B), 4(\mathrm{~cm}), 4(\mathrm{~cm})$ \\
\hline Wavelet bandwidth $B$, basis functions & $64(p x)$, Daubechies- $D 8$ \\
\hline Explored area & $1485\left(\mathrm{~m}^{2}\right)$ \\
\hline Number of classified objects & 31 \\
\hline Number of segmented object candidates & 1716 \\
\hline
\end{tabular}

Stand-alone evaluation Initially, we compare the discriminative power in using the proposed projective light diffusion image $N$ (abbreviated as PLDI) against a conventional depth image $\tilde{J}$ (abbreviated as Depth), while keeping identical all other components of our methodology. We use the recall performance measure, namely, the ratio of true positives to the sum of true positives and false negatives. The results are shown in the diagram of Fig. 5 for each object class together with the macro-averaged performance.

The performance benefit by using PLDI against depth images is evident across the majority of object classes with an overall gain in the order of $9.8 \%$. Interestingly, this result contrasts previous experiments in [16] (cf. page 11, Fig. 12) wherein depth images were consistently found superior to surface orientation images in the context of large-scale, content-based 3D model retrieval. We believe that the advantage of PLDI is mainly attributed to the fact that it is enriched with photometric information due to the use of JBF, as opposed to pure surface orientation images.

To evaluate this hypothesis, we tested performance by substituting the JBF with an equivalent (same kernel) bilateral filter (BF) which is applied to the raw depth image $J$ (abbreviated as PLDI-BF). From Fig. 5, we can attest that classification performance is indeed favoured through

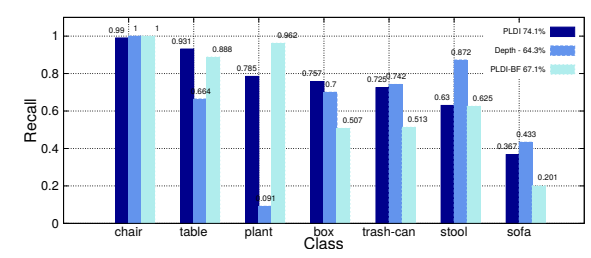

Figure 5. Performance comparison between proposed methodology (PLDI) against baseline alternatives (Depth, PLDI-BF) 
the application of JBF and the integration of photometric information in all classes except the plant class. Instances belonging to the sofa class were overall the most difficult to perceive due to their surface material which severely degraded depth acquisition from the sensor and propagated errors from reconstruction until shape description.

The overall superiority of PLDI is also consistently validated in terms of macroscopic precision (ratio of true positives to the sum of all positives), as given in Table III.

TABLE III. Macro-averaged precision comparison

\begin{tabular}{|l||c|c|c|}
\hline & PLDI & PDLF-BF & Depth \\
\hline Precision & $\mathbf{7 5 . 3} \%$ & $65 \%$ & $67.1 \%$ \\
\hline
\end{tabular}

In terms of efficiency, the overall framework enables real-time object classification using an optimized $\mathrm{C}++$ implementation on a contemporary computer (Intel i7, $3.40 \mathrm{GHz} \times 8$ ). Table IV reports the average frame rate in $\mathrm{Hz}$ attained for the complete pipeline from raw data processing until classification, together with the efficiency gain that is possible for different levels of parallelization via multithreading. Notably, real-time performance is feasible even without parallelization. In Table VI we report how processing time is on average partitioned among the main processing blocks of the entire pipeline. The most time-demanding part corresponds to the PLDI extraction due to the computation of the eigenvector decomposition of local patches across the entire point cloud. As expected, feature matching is extremely efficient (around $1 \mu s$ per comparison) due to its low dimensionality, that would still allow real-time queries from considerably bigger object repositories.

TABLE V. Timing ratios TABLE IV. Time performance RGB-D filtering $21 \%$

\begin{tabular}{|c|c|c|c|c|c|}
\hline & \multicolumn{3}{|c|}{ \# threads } & PLDI extraction & $38.6 \%$ \\
\hline & \begin{tabular}{|l|l}
1 & 2 \\
\end{tabular} & \begin{tabular}{l|l}
2 & 4
\end{tabular} & 6 & Clustering & $19.8 \%$ \\
\hline Frames/sec & \begin{tabular}{|l|l|}
6.2 & 6 \\
\end{tabular} & \begin{tabular}{|l|l}
5.97 .3 \\
\end{tabular} & 7.5 & Feature extraction & $20.5 \%$ \\
\hline & & & & Matching & $6 e-6 \%$ \\
\hline
\end{tabular}

Finally, examples of the qualitative performance from various scenes of the experiments are illustrated in Fig. 6. In a number of cases (e.g. table, plant and stool), it is worth noting that despite the relatively reduced intra-class object similarity, the proposed approach is successful in appointing the correct object label. The effect of strong exterior lighting can also be noticed (mainly for scenes \#2 and \#5) which was the most frequent cause of misclassification, nevertheless, the problem of missing depth can be significantly alleviated through the JBF application.

Comparison with other methods We have compared PLDI against state-of-the-art descriptors used in robotics and of computational efficiency similar to PLDI, namely,
TABLE VI. Comparative evaluation of descriptors

\begin{tabular}{l|llll}
\hline & Precision & Recall & Dimensionality & $P R_{\text {norm }}$ \\
\hline PLDI & $\mathbf{7 5 . 3}$ & $\mathbf{7 4 . 1}$ & $\mathbf{5 8}$ & $\mathbf{1 . 2 8 8}$ \\
ESF & 48.1 & 55.6 & 640 & 0.08 \\
VFH & 40.4 & 32.0 & 308 & 0.117 \\
SHOT & 36.6 & 38.9 & 704 & 0.053 \\
\hline
\end{tabular}

Viewpoint Feature Histogram (VFH) [24], Ensemble of Shape Functions (ESF) [25] and Signature of Histograms of Orientations (SHOT) [26]. We use the implementations provided in PointCloudLibrary (PCL) and the suggested settings for all methods according to [27], namely, we first voxelize a point cloud to a grid of $0.01 \mathrm{~cm}$ and set the radius for normal vector computation needed for VFH and SHOT to $0.03 \mathrm{~cm}$. Descriptors are extracted off-line for each method for the synthetic dataset in the same mode as for PLDI, while during on-line detection and classification, we adopt the same object clustering and segmentation as described in Sec. ?? and obtain object labels using the nearest neighbour. To obtain a global descriptor and perform matching using SHOT, we follow [27] (cf. Section II) which uses the average of the set of keypoints as global descriptor and then computes the sum of $L_{1}$ distances between centroids and their respective standard deviations.

In Table VI we show the comparative performance in terms of macro-averaged precision and recall. We further evaluate the normalized descriptiveness of each method by taking into account their dimensionality. Equivalently to the evaluation within [28] (cf eq. (6)), we take the ratio of average performance (here the average of precision and recall) to the total number of dimensions. This expresses the discriminative performance per descriptor dimension, which we denote as $P R_{\text {norm }}$. Clearly, the proposed PLDI method attains a considerable advantage against all compared methods while achieving this with the lowest dimensionality. We attribute the performance gain of PLDI to certain important aspects. Relatedly, while there exists visual resemblance between the synthetic objects and those encountered in reality, there is no exact correspondence and therefore descriptor variance between the two domains is high. The quantitative results suggest that PLDI can capture more effectively the discriminative object features compared to the other.

In addition, we believe that the other tested methods are not sufficiently robust to alleviate the extended range of sensor noise levels within the maximum permissible distance of our scenarios which is set to 4 meters and the considerably increased variation of sensing conditions due to exploration of different rooms. The fact that the average sensor-object distance within our experiments was 2.6 meters which is relatively higher than the implicitly assumed for the compared descriptors, shows that the PLDI can exploit more 


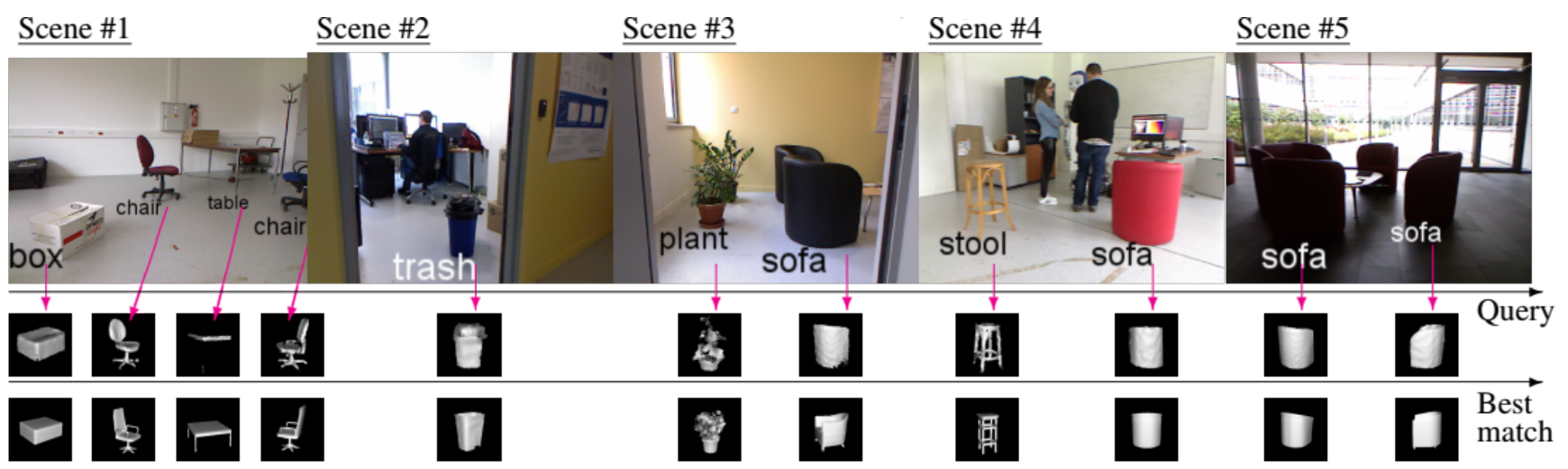

Figure 6. Qualitative classification results using data association. First row; Examples scenes. Middle row; corresponding reconstructed PLDI of segmented objects. Bottom row; corresponding nearest neighbours from the synthetic dataset.

TABLE VII. Evaluation in ModelNet10

\begin{tabular}{|c|c|c|c|c|c|c|c|c|c|c|c|}
\hline \multicolumn{11}{|c|}{ Precision \% } & \multirow[b]{2}{*}{$P_{\text {norm }}$} \\
\hline & bathtub & bed & chair & desk & dresser & monitor & $\begin{array}{l}\text { night } \\
\text { stand }\end{array}$ & sofa & table & toilet & \\
\hline PCD & 172.4 & 75.2 & 78.0 & 53.9 & 643 & 80.4 & 56.8 & 67.5 & 80.2 & 85.0 & 1.231 \\
\hline ESF & 78.9 & 74 & 80.5 & 57.5 & & & 60.9 & & & & \\
\hline & 73.0 & 75.9 & 77.5 & 49.9 & 63 & 84.7 & 54.3 & & & & \\
\hline & $\mathrm{T} 42.0$ & 48.6 & 53.3 & 43.6 & 55 & 55.1 & 38.3 & 49.1 & & & \\
\hline & & 51.6 & 64.8 & 47.2 & 60.8 & 64.1 & 52.1 & 49.9 & 80 & 70.5 & \\
\hline \multicolumn{12}{|c|}{ Recall \% } \\
\hline & bathtub & bed & chair & desk & dresser & monitor & $\begin{array}{l}\text { night } \\
\text { stand }\end{array}$ & sofa & table & toilet & $R_{n o}$ \\
\hline & 160.5 & 80. & 75. & 45. & & 2.2 & 54.0 & 76 & 79 & 89 & 1.222 \\
\hline & 68.0 & 75.7 & 76.3 & 54.3 & & 70.1 & 62.8 & & 79 & 84.9 & \\
\hline & 61.6 & 81.3 & 57.0 & 40.4 & 72.4 & 77.6 & 59.7 & 79.9 & 73.2 & 87.8 & 0.224 \\
\hline & 24.7 & 62.8 & 47.5 & 42.7 & 54 & 60.1 & 48.1 & 42.6 & 53.3 & 77.2 & 0.073 \\
\hline & $\$ 28.7$ & 59.0 & 55.5 & 47.4 & 60.2 & 68.8 & 55.2 & 50.5 & 71.6 & 80.1 & 0.214 \\
\hline
\end{tabular}

effectively the acquired sensory data.

We performed further experiments within the Princeton ModelNet10 [15] dataset, containing 4899 objects distributed into 10 common indoor classes. We also include another local descriptor called RoPS (Rotational Projection Statistics) which corresponds to the most informative local 3D shape descriptor according to [28], also available in PCL. ROPS was omitted from the previous experiment due to computational constraints that prevented its on-line exploitation. We use the default parameters while for descriptor matching we follow the same paradigm as with the SHOT local descriptor.

ModelNet10 is particularly suited for our purpose since the pose of each object coincides with its expected upright orientation. This allows us to adopt the same sensing configuration, namely, using $M=20$ camera viewpoints uniformly distributed around each object at a fixed sensor pose (this produces a total of $908 \times 20=18160$ descriptors for the entire test dataset). We perform the experiments by using each individual descriptor extracted from a viewpoint of a test object as a query and compare it to the viewpoint descriptors from the remaining set of test objects. Finally, we calculate the macro precision and recall performance and overall descriptiveness respectively, i.e. $P_{\text {norm }}$ and $R_{\text {norm }}$.
Table VII shows that the proposed PLDI descriptor exhibits the highest normalized descriptiveness $P_{\text {norm }}$ and $R_{n o r m}$ by a large margin. This shows its capacity in capturing informative object features both from real noisy sensory data as well as from noise-free synthetic data. On the other hand, in terms of absolute, non-normalized performance, ESF ranks first in most classes of this dataset followed by PLDI and the remaining methods. We attribute this to the ideal sensing conditions for extracting the descriptors, namely, the absence of light variability or sources of noise and the perfectly segmented object views, which was not the case in the real-world robotic experiment. Overall, the proposed descriptor proved considerably superior in the real-world experiment both in absolute as well as in normalized performance, consistently surpassed descriptors SHOT, ROPS and VFH in all experiments and showed comparable absolute performance with ESF in simulation.

\section{Conclusions}

We presented an original approach for real-time 3D object perception in indoor environments during robot exploration, on the basis of a novel, highly discriminative shape representation termed as projective light diffusion image expansion. Its advantage relies primarily on its capacity to encode both depth, surface orientation and photometric information within a concise representation that is robust to sensor noise and computationally efficient. The proposed PLDI advances the state-of-the-art of unsupervised object perception through its superior performance/dimensionality ratio in realistic as well as optimal sensing conditions.

\section{Acknowledgements}

This work has been funded by BpiFrance under the PSPC project Romeo 2. 


\section{References}

[1] T. Breuer, G. R. Giorgana Macedo, R. Hartanto, N. Hochgeschwender, D. Holz, F. Hegger, Z. Jin, C. Müller, J. Paulus, M. Reckhaus, J. A. Álvarez Ruiz, P. G. Plöger, and G. K. Kraetzschmar, "Johnny: An autonomous service robot for domestic environments," Journal of Intelligent \& Robotic Systems, vol. 66, no. 1, pp. 245-272, 2012.

[2] A. Aldoma, F. Tombari, J. Prankl, A. Richtsfeld, L. Di Stefano, and M. Vincze, "Multimodal cue integration through hypotheses verification for rgb-d object recognition and 6dof pose estimation," in Int. Conf. on Robotics and Automation, 2013.

[3] M. Gunther, T. Wiemann, S. Albrecht, and J. Hertzberg, "Model-based furniture recognition for building semantic object maps," Artificial Intelligence, 2015.

[4] J. R. Siddiqui, H. Andreasson, D. Driankov, and A. J. Lilienthal, "Towards visual mapping in industrial environments a heterogeneous task-specific and saliency driven approach," in IEEE Int. Conf. on Robotics and Automation, 2016.

[5] E. Martinez-Martin and A. P. del Pobil, "Object detection and recognition for assistive robots," IEEE Robotics Automation Magazine, vol. PP, no. 99, pp. 1-1, 2017.

[6] S. Song and J. Xiao, "Sliding shapes for 3d object detection in depth images," in European Conf. in Computer Vision, 2014.

[7] S. Gupta, R. Girshick, P. Arbeláez, and J. Malik, "Learning rich features from rgb-d images for object detection and segmentation," in European Conf. on Computer Vision, 2014.

[8] B.-S. Hua, Q.-T. Truong, M.-K. Tran, Q.-H. Pham, A. Kanezaki, T. Lee, H. Chiang, W. Hsu, B. Li, Y. Lu, H. Johan, S. Tashiro, M. Aono, M.-T. Tran, V.-K. Pham, H.-D. Nguyen, V.-T. Nguyen, Q.-T. Tran, T. V. Phan, B. Truong, M. N. Do, A.-D. Duong, L.-F. Yu, D. T. Nguyen, and S.-K. Yeung, "RGB-D to CAD Retrieval with ObjectNN Dataset," in Eurographics Workshop on 3D Object Retrieval, 2017.

[9] L.-C. Caron, D. Filliat, and A. Gepperth, "Neural network fusion of color, depth and location for object instance recognition on a mobile robot," in ECCV Workshop, 2014.

[10] A. Anand, H. S. Koppula, T. Joachims, and A. Saxena, "Contextually guided semantic labeling and search for threedimensional point clouds," Int. J. Rob. Res., vol. 32, no. 1, pp. 19-34, 2013.

[11] D. Pangercic, B. Pitzer, M. Tenorth, and M. Beetz, "Semantic object maps for robotic housework - representation, acquisition and use," in Int. Conf. on Intelligent Robots and Systems, 2012.

[12] H. Su, S. Maji, E. Kalogerakis, and E. G. Learned-Miller, "Multi-view convolutional neural networks for 3d shape recognition," in Int. Conf. on Computer Vision, 2015.

[13] W. J. Beksi and N. Papanikolopoulos, "Object classification using dictionary learning and rgb-d covariance descriptors," in Int. Conf. on Robotics and Automation, 2015.
[14] M. Blum, J. Springenberg, J. Wulfing, and M. Riedmiller, "A learned feature descriptor for object recognition in rgb-d data," in Int. Conf. on Robotics and Automation, 2012.

[15] Z. Wu, S. Song, A. Khosla, F. Yu, L. Zhang, X. Tang, and J. Xiao, "3d shapenets: A deep representation for volumetric shapes," in Conf. on Computer Vision and Pattern Recognition, 2015.

[16] P. Papadakis, I. Pratikakis, T. Theoharis, and S. Perantonis, "Panorama: A 3d shape descriptor based on panoramic views for unsupervised 3d object retrieval," Int. Journal of Computer Vision, vol. 89, no. 2-3, pp. 177-192, 2010.

[17] K. Sfikas, T. Theoharis, and I. Pratikakis, "Exploiting the PANORAMA Representation for Convolutional Neural Network Classification and Retrieval," in Eurographics Workshop on 3D Object Retrieval, 2017.

[18] K. Khoshelham and S. O. Elberink, "Accuracy and resolution of kinect depth data for indoor mapping applications," Sensors, vol. 12, no. 2, pp. 1437-1454, 2012.

[19] E. Eisemann and F. Durand, "Flash photography enhancement via intrinsic relighting," in ACM SIGGRAPH, 2004.

[20] E. S. L. Gastal and M. M. Oliveira, "Domain transform for edge-aware image and video processing," in ACM SIGGRAPH, 2011.

[21] S.-Y. Kim, J.-H. Cho, A. Koschan, and M. Abidi, "Spatial and temporal enhancement of depth images captured by a time-of-flight depth sensor," in Int. Conf. on Pattern Recognition, 2010.

[22] L. Chen, H. Lin, and S. Li, "Depth image enhancement for kinect using region growing and bilateral filter," in Int. Conf. on Pattern Recognition, 2012.

[23] A. Kumar Pandey, R. Gelin, R. Alami, R. Viry, A. Buendia, R. Meertens, M. Chetouani, L. Devillers, M. Tahon, D. Filliat, Y. Grenier, M. Maazaoui, A. Kheddar, F. Lerasle, and L. Fitte-Duval, "Romeo2 project: Humanoid robot assistant and companion for everyday life: I. situation assessment for social intelligence," in International Workshop on Artificial Intelligence and Cognition, CEUR Workshop Proceedings, 2014.

[24] R. Rusu, G. Bradski, R. Thibaux, and J. Hsu, "Fast 3d recognition and pose using the viewpoint feature histogram," in Int. Conf. on Intelligent Robots and Systems, 2010.

[25] W. Wohlkinger and M. Vincze, "Ensemble of shape functions for $3 \mathrm{~d}$ object classification," in Int. Conf. on Robotics and Biomimetics, 2011.

[26] F. Tombari, S. Salti, and L. Stefano, "Unique signatures of histograms for local surface description," in ECCV, 2010.

[27] L. A. Alexandre, "3D descriptors for object and category recognition: a comparative evaluation," in Workshop on Color-Depth Camera Fusion in Robotics at the Int. Conf. on Intelligent Robots and Systems, 2012.

[28] Y. Guo, M. Bennamoun, F. Sohel, M. Lu, J. Wan, and N. M. Kwok, "A comprehensive performance evaluation of $3 \mathrm{~d}$ local feature descriptors," Int. Journal of Computer Vision, vol. 116, no. 1, pp. 66-89, 2016. 